\title{
Belowground Plant-Plant Interaction by Common Mycorrhizal Network (CMN)
}

\author{
B.R. Vanishri ${ }^{1 *}$ and Mamata Khandappagol ${ }^{2}$ \\ ${ }^{1}$ Department of Plant Biotechnology, UAS, GKVK, Bangalore 560065, Karnataka, India \\ ${ }^{2}$ Department of Genetics \& Plant Breeding, UAS, GKVK, Bangalore 560065, Karnataka, India \\ *Corresponding author
}

\section{A B S T R A C T}

\section{Keywords \\ Common mycorrhizal network, Interplant communication, Defence signals \\ Article Info \\ Accepted: \\ 12 August 2018 Available Online: 10 September 2018}

We all know that Plants are sessile organisms they cannot go and talk like us, but plants continuously interact with each other and share their environment by different ways of communication. One of the important ways of interplant communication is belowground communication through Common Mycorrhizal Network, Plants are so kind they care and share each other, they use Common Mycorrhizal Network for sharing the nutrients and defence signals. Few intelligent plants hijack CMN to transfer allelochemicals. Plants are so generous they reward the fungi with carbohydrates and help for its survival. This interplant signal transfer and response is efficiently helpful in many plants to combat herbivore/abiotic stress. This interplant communication can be effectively used in agriculture to get higher productivity.

\section{Introduction}

We humans live a social life, we communicate to each other by various ways; we use lots of applications and devices for communication (Fig. 1).

We interact regularly through social media, we talk to each other and we share our feelings.

Like us plants are living beings, they are not simply sitting and quietly growing, they also have social life, they interact regularly with each other but the way of communication/ interaction and media used for communication is different.

\section{Can plants talk?}

The answer is YES, but not like humans, plants developed their own way of communication systems.

In nature, plants are sessile organisms they cannot go and talk like us but plants continuously interact with other living organisms that share their environment/ physiological status. Plants communications are mainly by two ways i.e., aboveground and belowground communications. In aboveground communication plants use volatile organic compounds (VOCs) as their media to interact with other living organisms that includes plants, pests, pollinators and 
other beneficial insects. Different volatiles will be used for communication with different organism. In this paper we are not discussing about aboveground air borne signalling.

In case of belowground communication plant uses fungi i.e. Common mychorrhizal network (CMNs) for communication. Plants use fungi as a media for communication.

Plants talk to each other using an internet of fungus. Fungus is known as earth's natural internet. Most land plants associate with mycorrhizal fungi that can connect roots of neighbouring plants in Common Mycorrhizal Networks (CMNs), which is an information superhighway that speeds up interactions between a large, diverse population of individuals. It allows distant individuals to communicate and help each other out (Gilbert and Johnson, 2017) (Fig. 2, 4 and 11).

\section{CMN: Wood wide web}

In our world we have www i.e. World Wide Web for our networking, like that plants have wood wide web, it connects large number of plants to greater distances especially in forests. Plants recognize their relatives and share nutrients; basically tree families help each other out. Like that through CMN plants share so many informations. By linking to the fungal network they can help out their neighbours by sharing nutrients and information - or sabotage unwelcome plants by spreading toxic chemicals through the network (allelopathy) (Fig. 3).

\section{Relationship between fungi and plant}

Around $90 \%$ of land plants are in mutuallybeneficial relationships with fungi. In mycorrhizal associations, plants provide fungi with food in the form of carbohydrates. In exchange, the fungi help the plants suck up water, and provide nutrients like phosphorus and nitrogen, via their mycelia. Since the 1960s, it has been clear that mycorrhizae help individual plants to grow.

Fungal networks also boost their host plants' immune systems. That's because, when a fungus colonises the roots of a plant, it triggers the production of defence-related chemicals. These make later immune system responses quicker and more efficient, a phenomenon called "priming". Simply plugging in to mycelial networks makes plants more resistant to disease.

\section{Mycorrhizal fungi}

Myco means Fungi and Rhizza means Roots, the 19th-century German biologist Albert Bernard Frank coined the word "mycorrhiza" to describe the symbiotic partnerships, in which the fungus colonises the roots of the plant.

There are three broad categories of mycorrhizal fungi; Ectomycorrhizal (ECM) fungi, Ericoid mycorrhizal (ERM) fungi and Arbuscular mycorrhizal (AM) fungi and these are likely to differ in the extent and function of their CMNs. Most AM and ECM fungi (with the exception of smooth contact type) produce extraradical mycelium and some 'long-distance' growth forms produce rhizomorphs that can grow many metres through soil giving them potential to connect many roots (Taylor, 2006). ERM fungi tend to have hyphae that are concentrated close to their hosts' roots, so CMNs formed by ERM fungi are likely to operate only at a localized spatial scale (Grelet et al., 2010).

The length of extraradical mycelia of AM and ECM fungi vary considerably, with typical estimates ranging from 10 to $100 \mathrm{~m}$ hyphae /g soil, or even up to hundreds of metres of hyphae /metre of root length (Leake et al., 2004). 


\section{Evidence of interplant signalling via $\mathrm{CMN}$}

The idea that CMNs could potentially send signals between plants has developed over the last few years due to various pieces of accruing evidence. For example, it is known that CMNs can transport mineral nutrients and other resources such as carbon (Simard et al., 1997) between plants. Since fungal hyphae can transfer large molecules such as lipids, amino acids and sugars (Bago et al., 2002), it therefore seems reasonable to question whether they may also be able to transport chemicals that elicit plant defences against pests and pathogens.

Further evidence had shown that semiochemicals can indeed move through liquids between hydroponically grown plants, thus stimulating receiving plants to elicit defences (Chamberlain et al., 2001). The mechanism for this was unknown and no CMNs were present; however, based on combining the above evidence of nutrient transfer via CMNs and semiochemical transfer through liquid, Dicke and Dijkman (2001) suggested that CMNs may be able to act as conduits for transfer of semiochemicals between connected plants (Fig. 6 and 7).

Interplant resource exchanges are regulated by source-sink relationships within the MN (Mycorrhizal network), where one plant that is rich in nutrients serves as a source (donor) of compounds for a neighbouring plant that is poor in nutrients, which thus serves as a sink (receiver) (Simard et al., 2012). Defense signals and allelochemicals also transported through MN. The long-distance transport of carbon and/or nutrients appears to occur predominantly by advective mass flow driven by the source-sink gradient generated by these interplant nutrient differences.

We now know that MNs can influence plant establishment, survival, physiology, growth and defence chemistry (Babikova et al., 2013). This influence is thought to occur because the MN serves either as a pathway for interplant exchange of resources and stress molecules or as a source of fungal inoculums.

\section{Do plants care \& share?}

Yes, plants share and care by supplying their nutrients to neighbours and young ones. In 1997, Suzanne Simard of the University of British Columbia showed large trees help out small, younger ones using the fungal internet. Without this help, many seedlings wouldn't survive.

Suzanne Simard also showed that Douglas fir and paper birch trees can transfer carbon between them via mycelia. Others have since shown that plants can exchange nitrogen and phosphorus as well, by the same route. In the 1997 study, seedlings in the shade - which are likely to be short of food - got more carbon from donor trees.

There are plants that don't have chlorophyll, so unlike most plants they cannot produce their own energy through photosynthesis. Some of these plants, such as the phantom orchid, get the carbon they need from nearby trees, via the mycelia of fungi that both are connected to (Fig. 8). Other orchids only steal when it suits them. These "mixotrophs" can carry out photosynthesis, but they also "steal" carbon from other plants using the fungal network that links them.

Plants also care their neighbours that mean when there is threat/ attack from pests plant gives warning signals to neighbouring plants. In figure 9, we can see Herbivores induce systemic defence response in the infested plant leading to emission of protective volatiles that repel subsequent herbivores from the plant and also attract their natural enemies. A signal is transferred via the $\mathrm{CMN}$ to a neighbouring 
plant to induce a similar defence response. The signal might be transferred further to an indirectly interconnected second neighbouring plant, and that it might "prime" that plant for potential future attack. Primed plants do not exhibit increased defence response; however, they respond more strongly and faster, if the attack occurs (Fig. 10).

\section{Warning signals}

In response to herbivore attack, plants activate a wide array of defences that can reduce herbivore damage, including blends of volatile organic compounds (VOCs) that can be used as foraging cues by natural enemies of the herbivores or VOCs may repel the Herbivore. Herbivore induced plant volatiles (HIPVs) have also been implicated in plant-plant communication, as they can be perceived by neighbouring plants and prime them for an enhanced response upon subsequent insect attack. By targeting jasmonic acid (JA)inducible genes, HIPVs have been shown to enhance both direct and indirect defence responses which can benefit the receiver by decreasing herbivore damage. In Belowground communication plants uses mycorrhizal fungi to transfer the warning signals to neighbouring plants.

\section{Evidences for defense signal transfer via CMN}

The first study to demonstrate CMN-based interplant signals used a biotrophic fungus (Alternaria solani) pathogenic on tomato plants that were grown with an AM fungus. To compare connected versus unconnected tomato plants, Song et al., (2010) used waterproof membranes between mycorrhizal 'donor' plants (those that received the pathogenic fungus) and uninfested receivers that completely prevented diffusion or made contrasts between donor and receiver plants that were both grown in the non-mycorrhizal condition. They therefore did not overcome all the experimental challenges listed above, such as teasing apart the effects exudation by mycorrhizal hyphae and diffusion of molecules through soil solution, but nevertheless showed striking results of disease resistance and activities of defensive enzymes in healthy neighbouring 'receiver' tomato plants when they were connected to an infested 'donor' tomato plant via a CMN. The subsequent study to demonstrate CMN-based interplant signals used aphids infesting bean plants, all of which were grown with an AM fungus (Babikova et al., 2013).

All bean plants were grown in the mycorrhizal state, and Babikova et al., (2013) were able to compare connected and non-connected plants in two ways that controlled for root-root contact and soil diffusion. A central donor bean plant that received aphids was surrounded by four 'receiver' bean plants (that never received aphids), two of which were connected to the donor via a CMN, and two of which were in the 'unconnected' treatment group. Of particular novelty was the use of two 'unconnected' treatment groups: the first was to simply prevent contact using a $0.5 \mathrm{~mm}$ mesh through which fungal hyphae cannot grow, and a second 'unconnected' treatment grew the receiver plant inside a $40 \mathrm{~mm}$ mesh core that did allow hyphal contact and, when aphids were added to the donor plant, this core was rotated to snap the hyphal connections.

The above-ground parts of all plants were sealed in plastic bags when aphids were added to the donor, to prevent any aerial communication. Babikova et al., (2013) analyzed the VOCs and measured the attractiveness of the VOCs to aphids and parasitoid wasps (aphid enemies) of all plants. They found that connected receiver plants produced the same concoction of VOCs as the aphid-infested donors, even though they themselves were not infested, as well as being 
similarly repellent to aphids and attractive to parasitoid wasps. By contrast, unconnected beans maintained the same VOC composition as expected from uninfested plants, and were attractive to aphids and unattractive to parasitoids, which is the normal state of plants that have not induced their defences. This demonstrated clearly and unequivocally that CMNs can be conduits for aphid-warning signals between plants, and redefines our understanding of multitrophic interactions.

\section{All plants are not good}

Plants have to compete with their neighbours for resources like water and light. As part of that battle, some release chemicals that harm their rivals. This "allelopathy" is quite common in trees, including acacias, sugarberries, American sycamores and several species of Eucalyptus. They release substances that either reduce the chances of other plants becoming established nearby, or reduce the spread of microbes around their roots.

Sceptical scientists doubt that allelopathy helps these unfriendly plants much. Surely, they say, the harmful chemicals would be absorbed by soil, or broken down by microbes, before they could travel far. But maybe plants can get around this problem, by harnessing underground fungal networks that cover greater distances. One of the beststudied examples of allelopathy is the American black walnut tree. It inhibits the growth of many plants, including staples like potatoes and cucumbers, by releasing a chemical called jugalone from its leaves and roots. Achatz and her team placed pots around walnut trees, some of which fungal networks could penetrate. Those pots contained almost four times more jugalone than pots that were rotated to keep out fungal connections. The roots of tomato seedlings planted in the jugalone-rich soil weighed on average $36 \%$ less.
Barto et al., 2011 grew golden marigolds in containers with mycorrhizal fungi. The pots contained cylinders surrounded by a mesh, with holes small enough to keep roots out but large enough to let in mycelia. Half of these cylinders were turned regularly to stop fungal networks growing in them. The team tested the soil in the cylinders for two compounds made by the marigolds, which can slow the growth of other plants and kill nematode worms. In the cylinders where the fungi were allowed to grow, levels of the two compounds were $179 \%$ and $278 \%$ higher than in cylinders without fungi.

That suggests the mycelia really did transport the toxins. The team then grew lettuce seedlings in the soil from both sets of containers. After 25 days, those grown in the more toxin-rich soil weighed $40 \%$ less than those in soil isolated from the mycelia. "These experiments show the fungal networks can transport these chemicals in high enough concentrations to affect plant growth," says Morris, who is now based at Xavier University in Cincinnati, Ohio.

\section{Why fungus helps plant in communication?}

Because fungus wants to protect its source of carbon.

Plants that receive a warning signal of aphid attack produce volatiles that repel aphids and attract parasitoids (that kill aphids) and so can clearly benefit from this signal. Signals from CMNs are likely to be of most importance between plants that are attacked by the same insect herbivore and that produce and detect the same volatiles, such as plants of the same family (e.g. the pea aphid and broad beans). However, what do AM fungi gain from transferring signals between plants? AM fungi rely on their plant hosts for carbon, essential for growth and most aspects of their functioning. 
Fig.1 Various social media applications used by humans

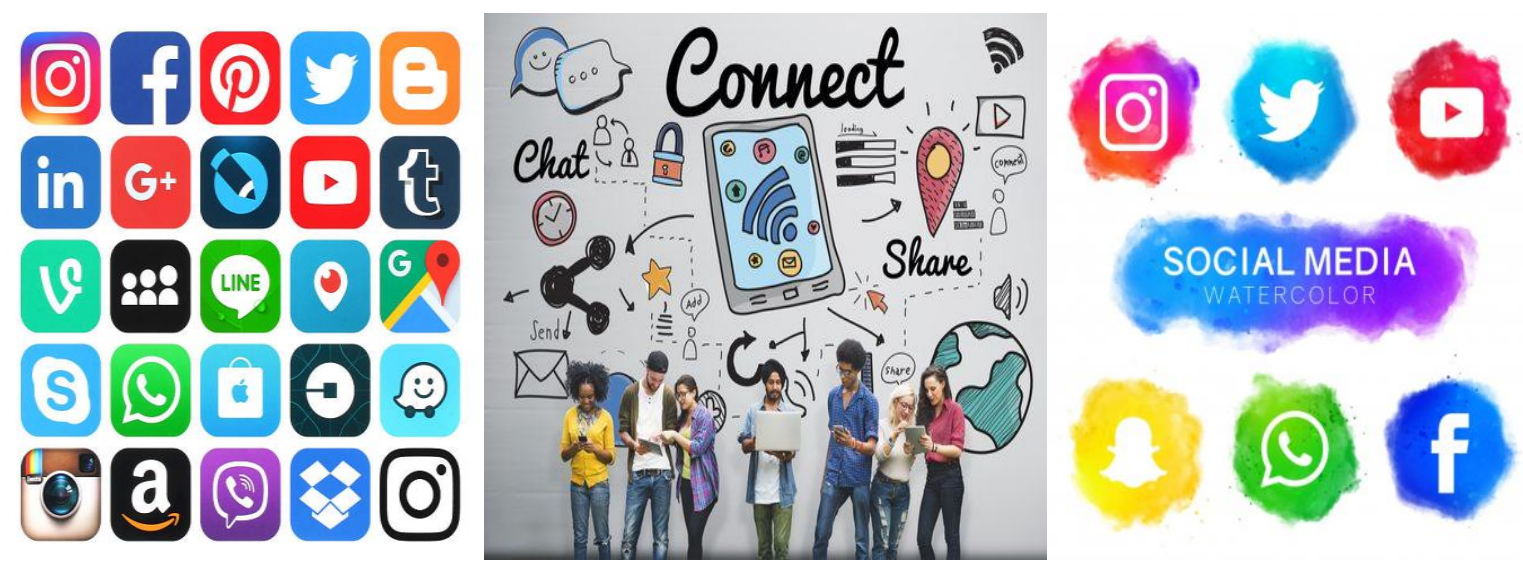

Fig.2 Ways of interplant communication
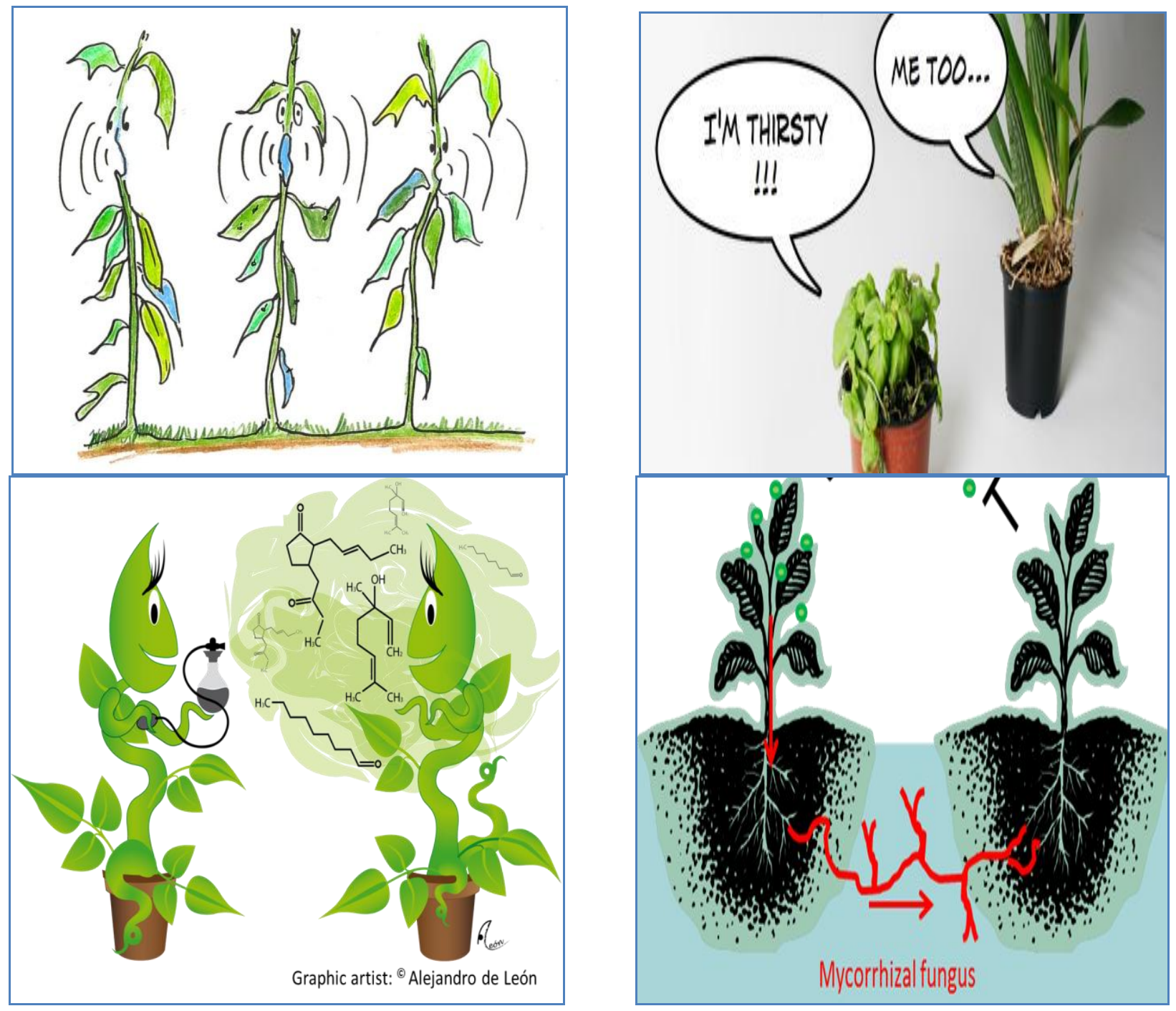
Fig.3 World Wide Web

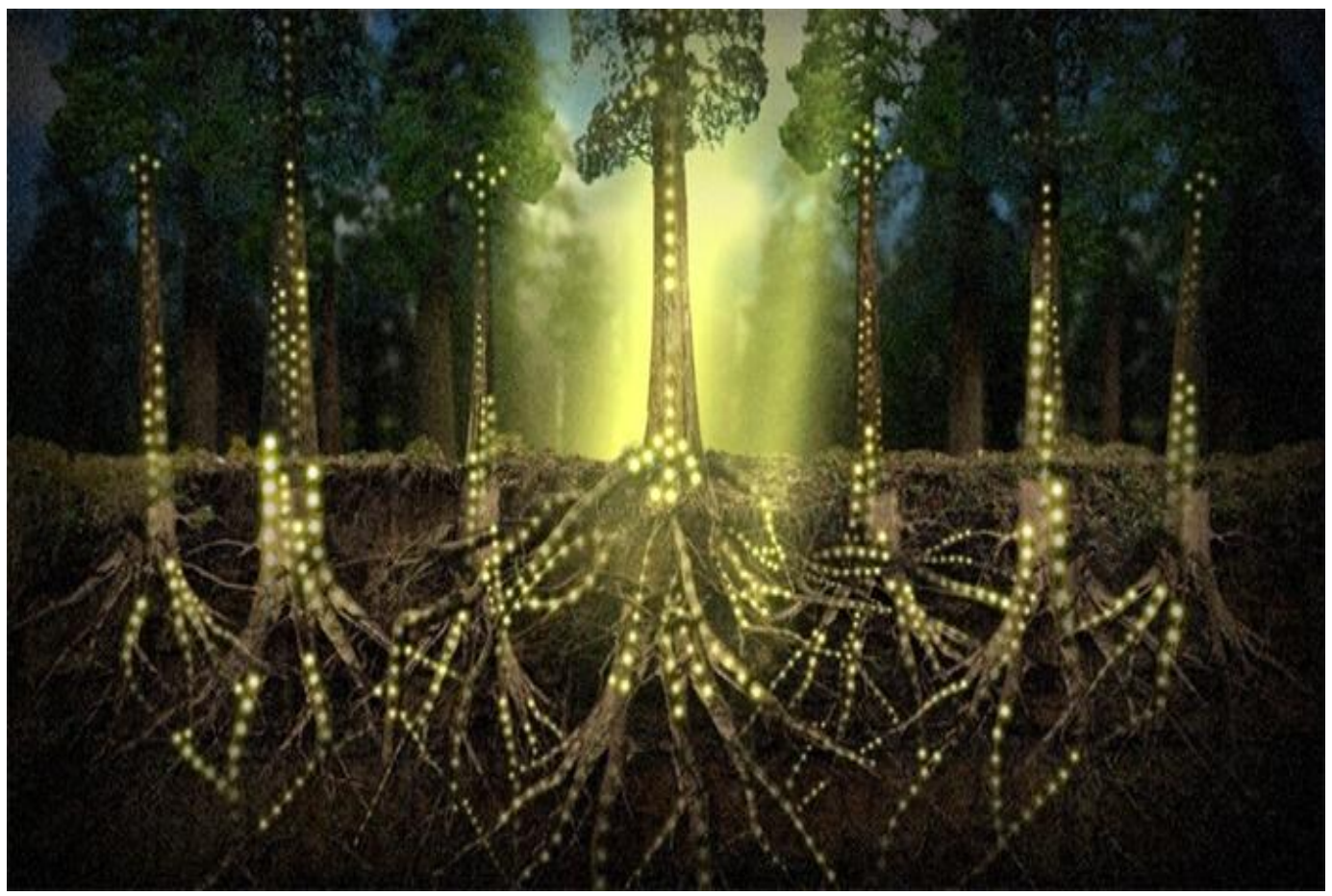

Fig.4 Mycorrhizal network between plants

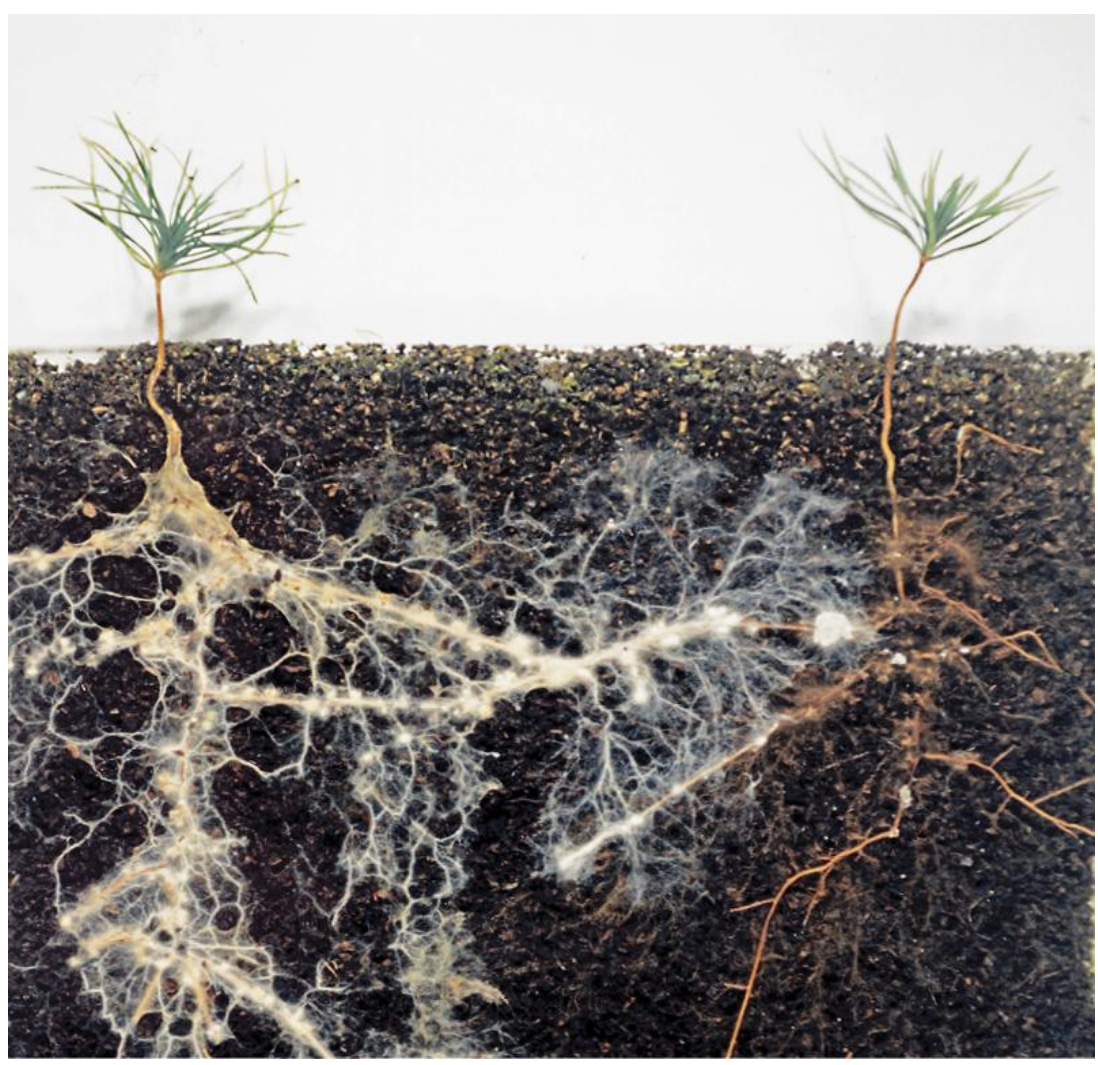


Fig.5 Schematic of resources and signals documented to travel through a CMN

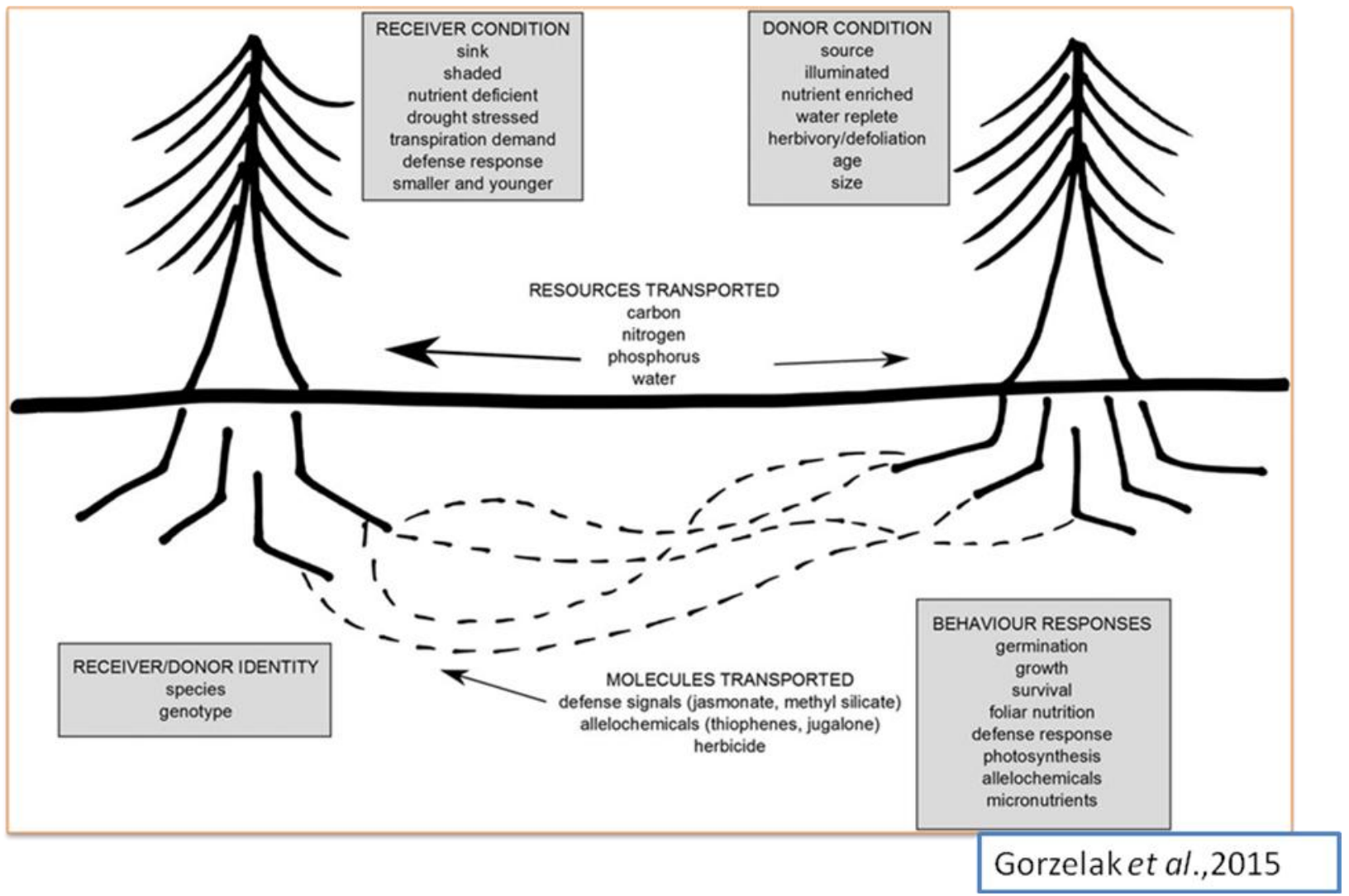

Fig.6 Nutrient transfer between plants

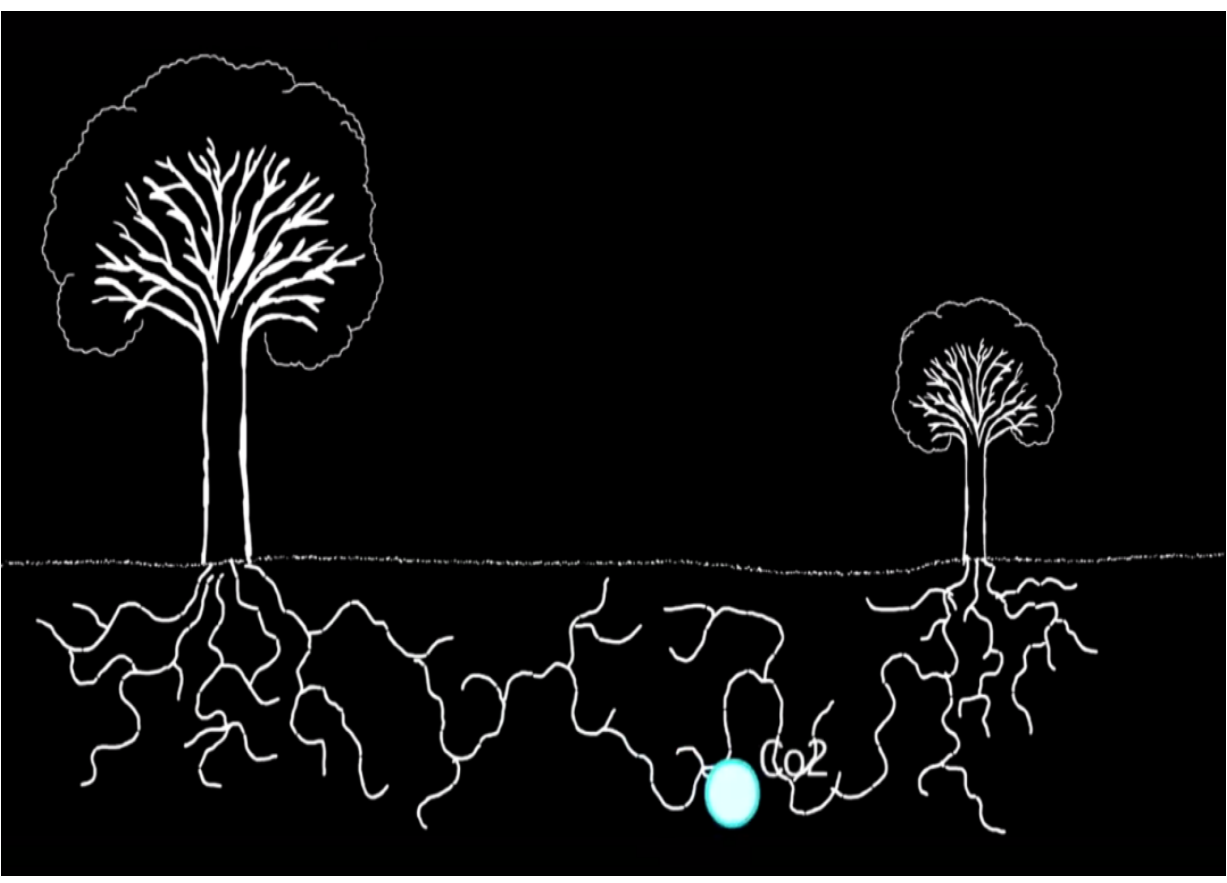


Fig.7 Nutrient transfer between douglas fir and paper birch
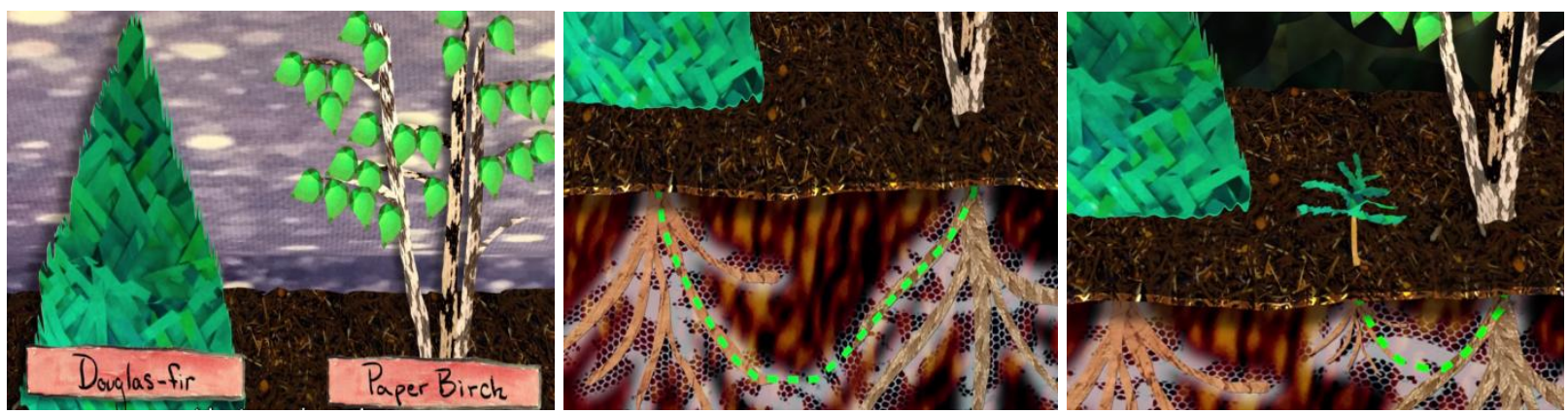

Fig.8 Phantom orchid

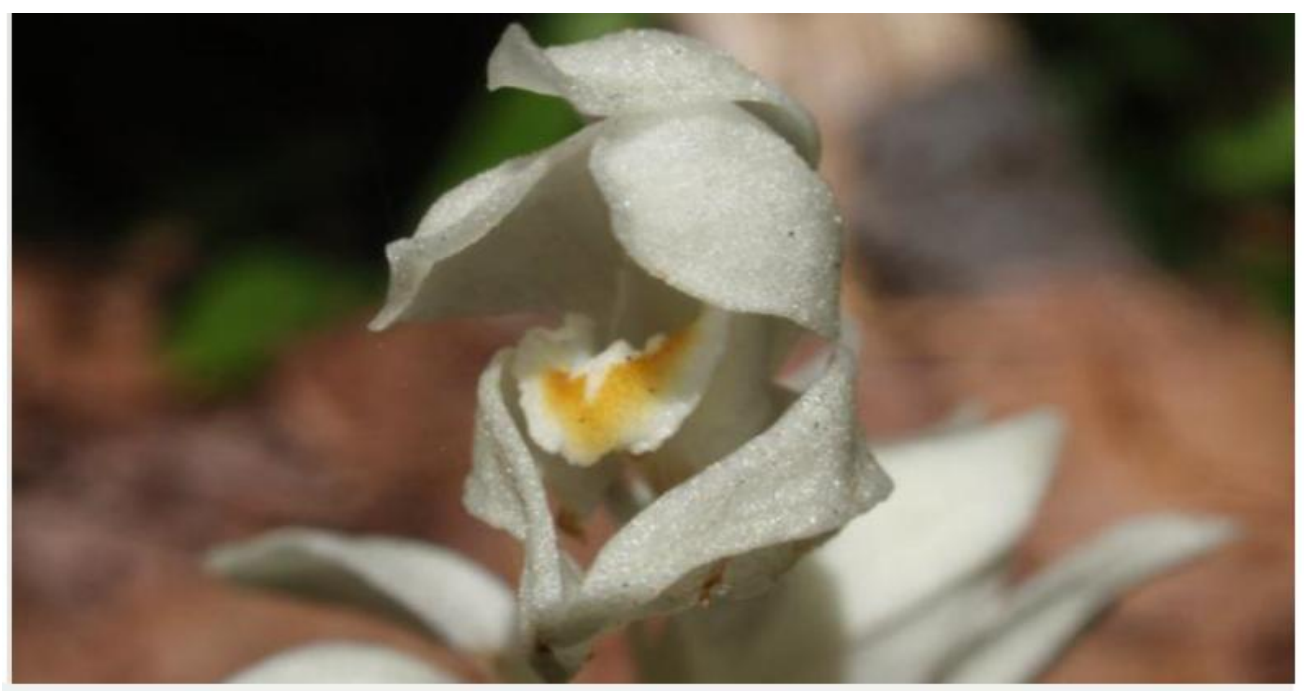

A phantom orchid (Cephalanthera austiniae) (Credit: Tom Hilton, CC by 2.0)

Fig.9 Defence-related communication between plants via a CMN
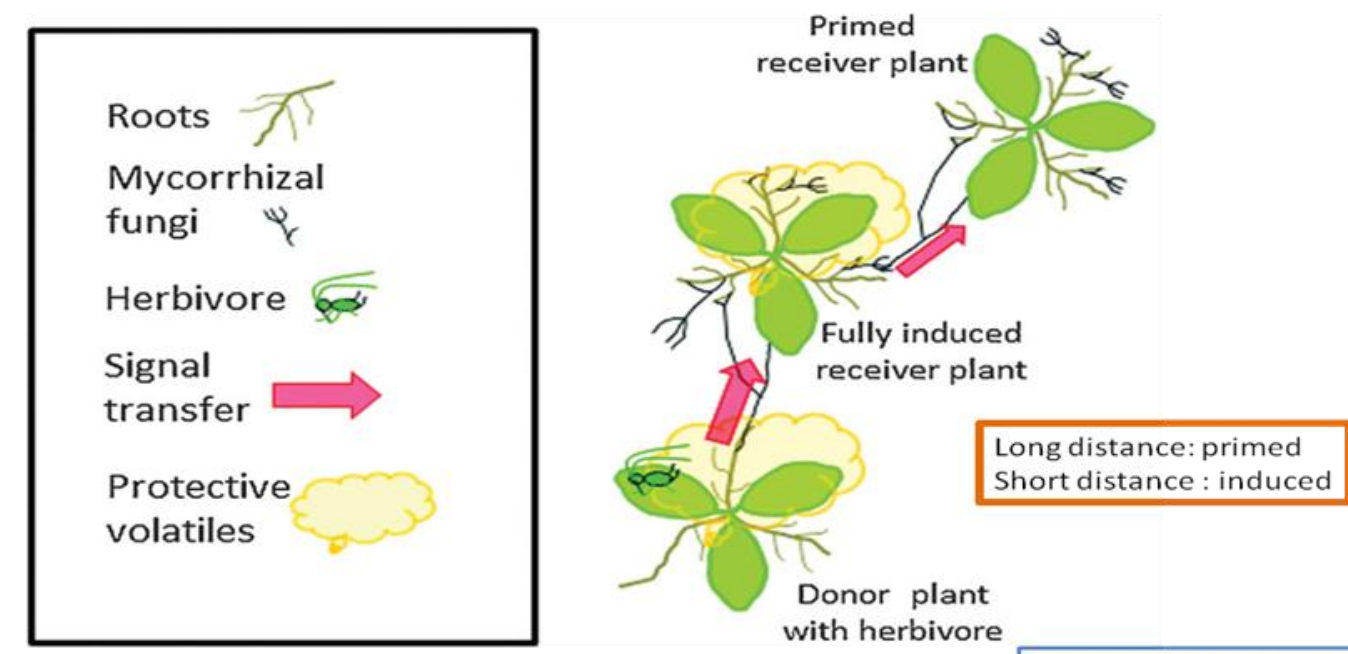

(Babikova et al., 2013) 
Fig.10 Plant-plant communication in response to herbivore attack

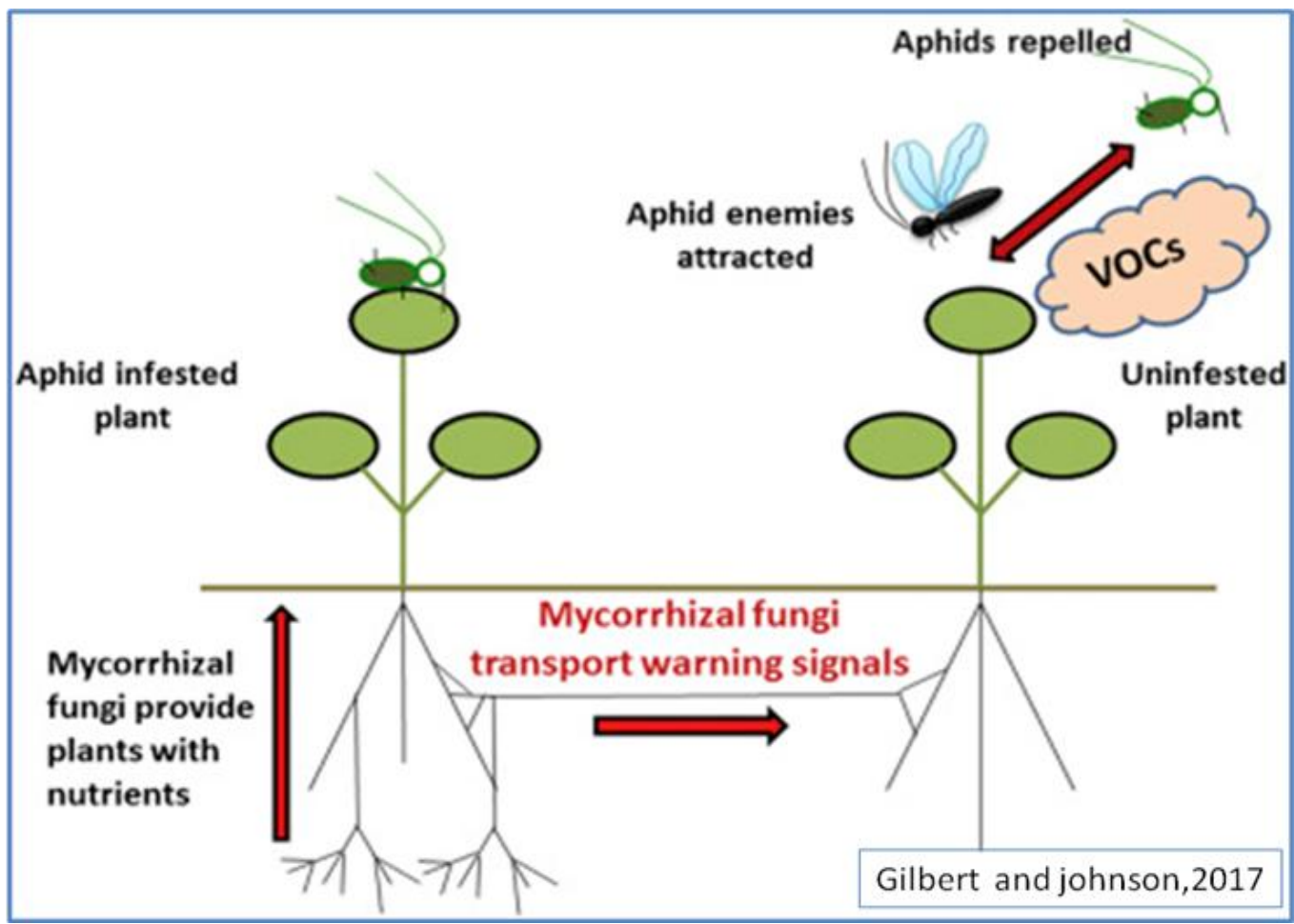

Fig.11 Mycorrhizal network between plants

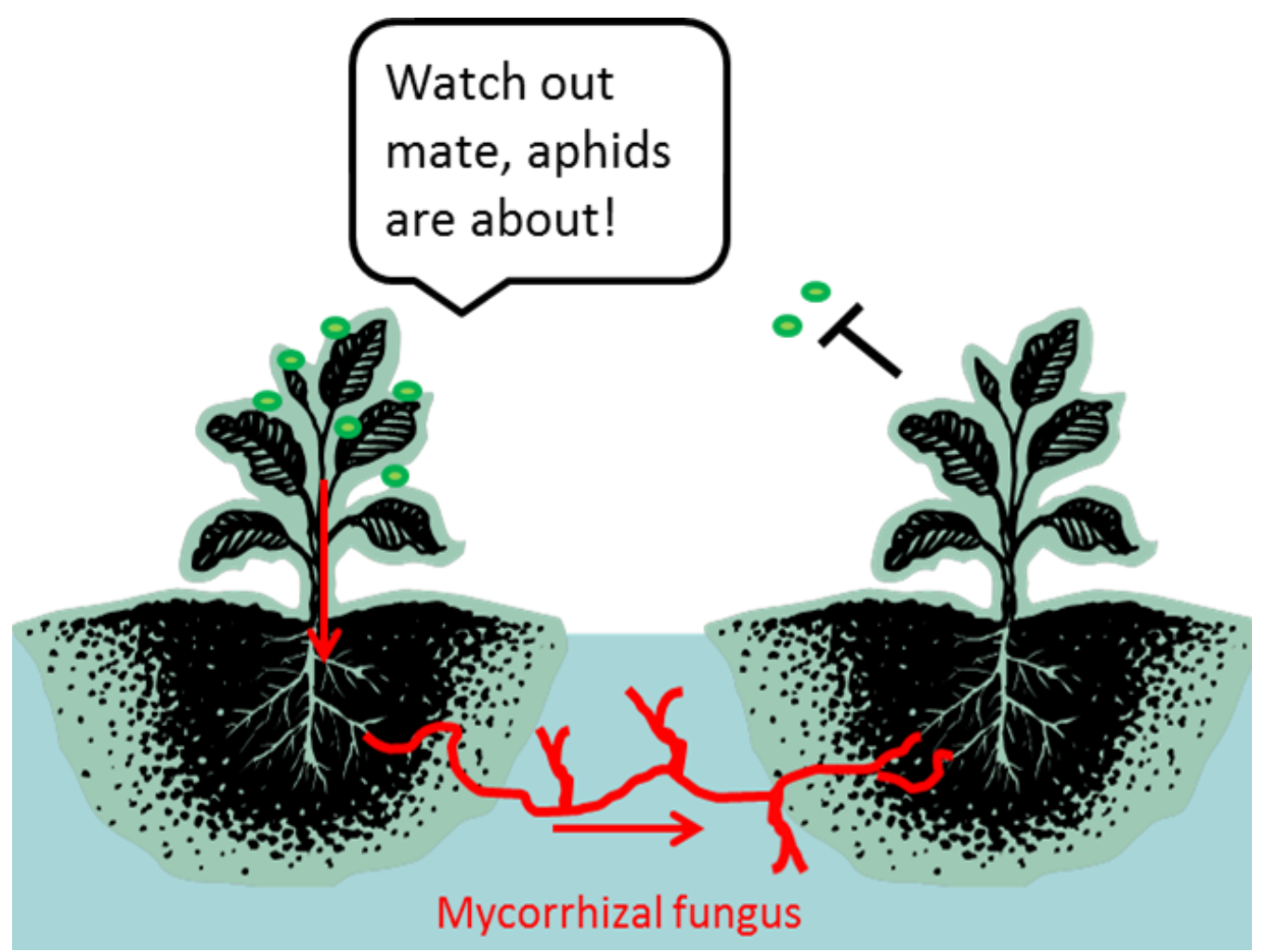


Fig.12 Signal transfer via a CMN is modulated in order to acquire the most benefit for the fungus

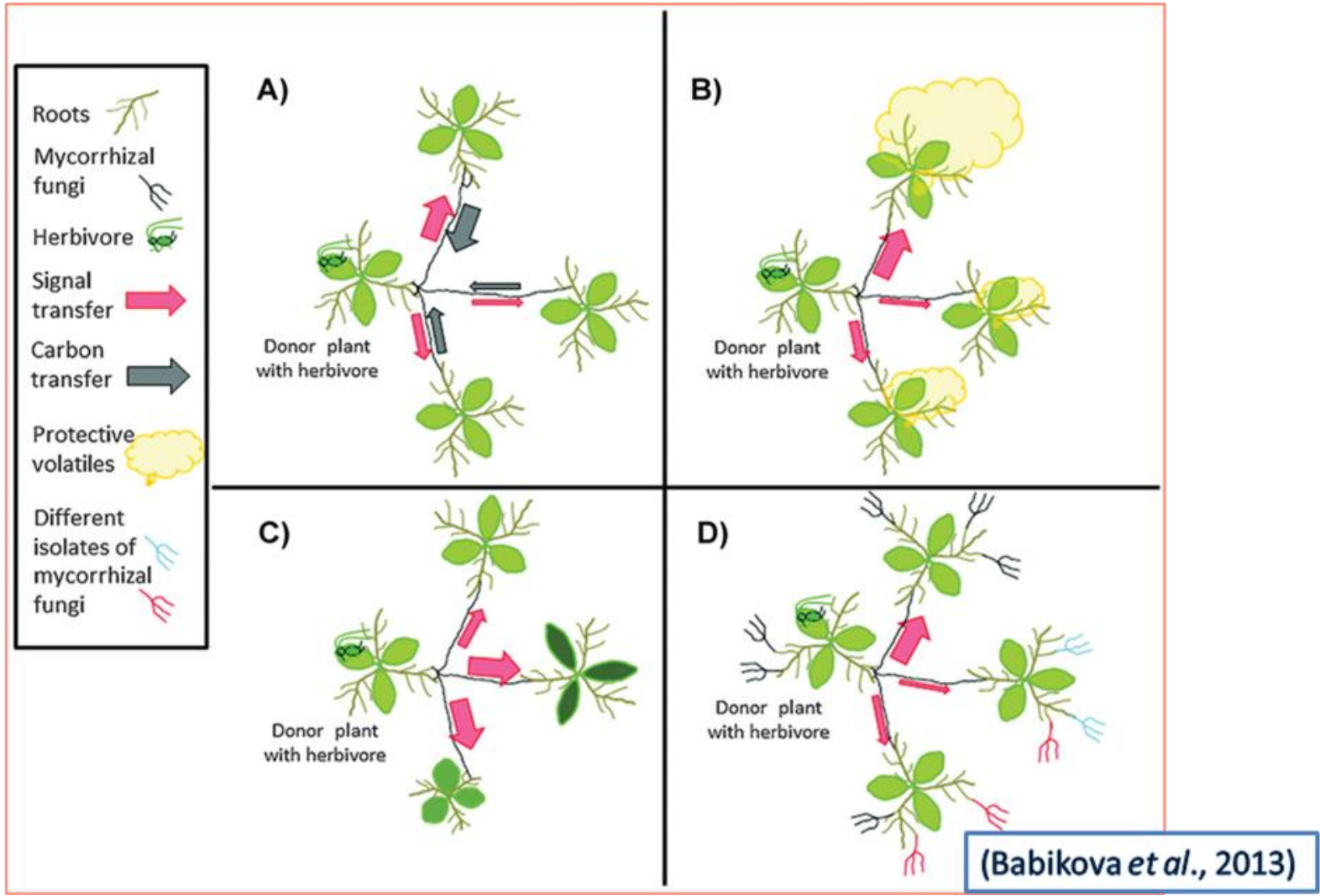

Fig.13 The speed of response of uninfested 'receiver' plants when connected via CMN to neighbouring plants infested with different pests or damaged mechanically

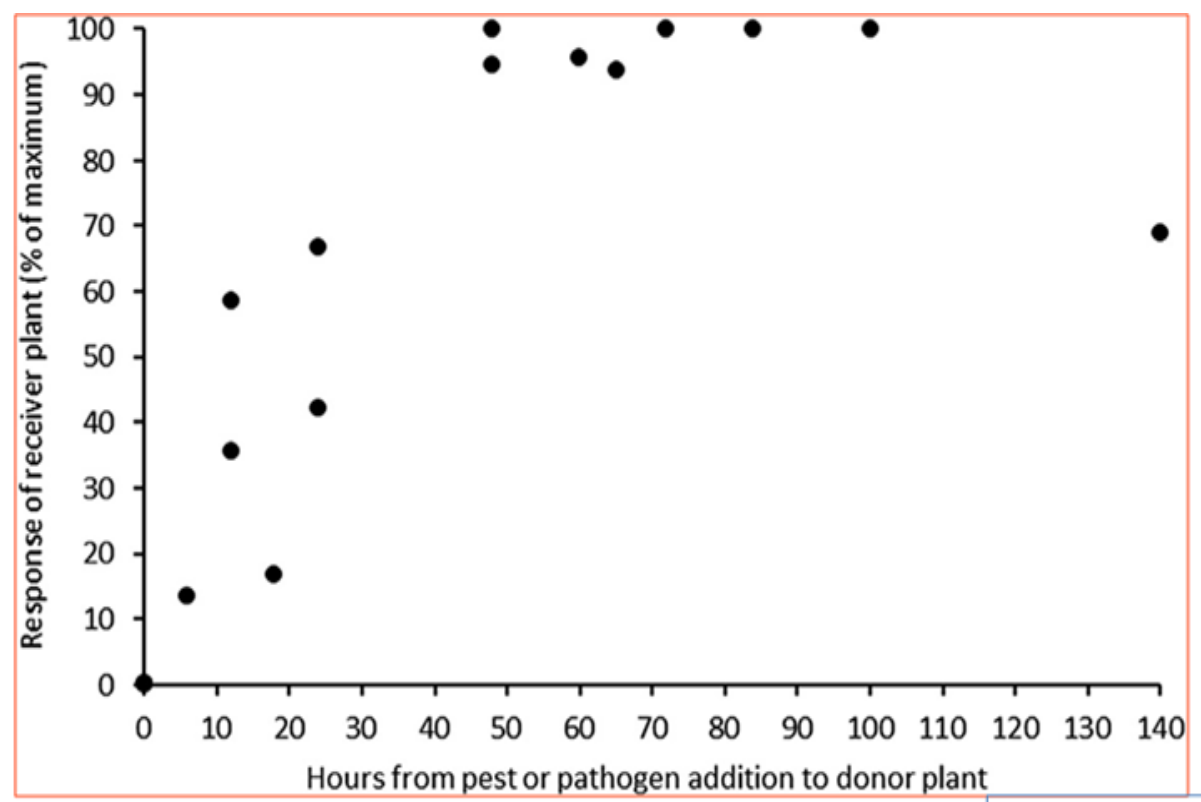

Gilbert and johnson,2017 
Fig.14 Impact of a CMN in monocultures and mixed culture

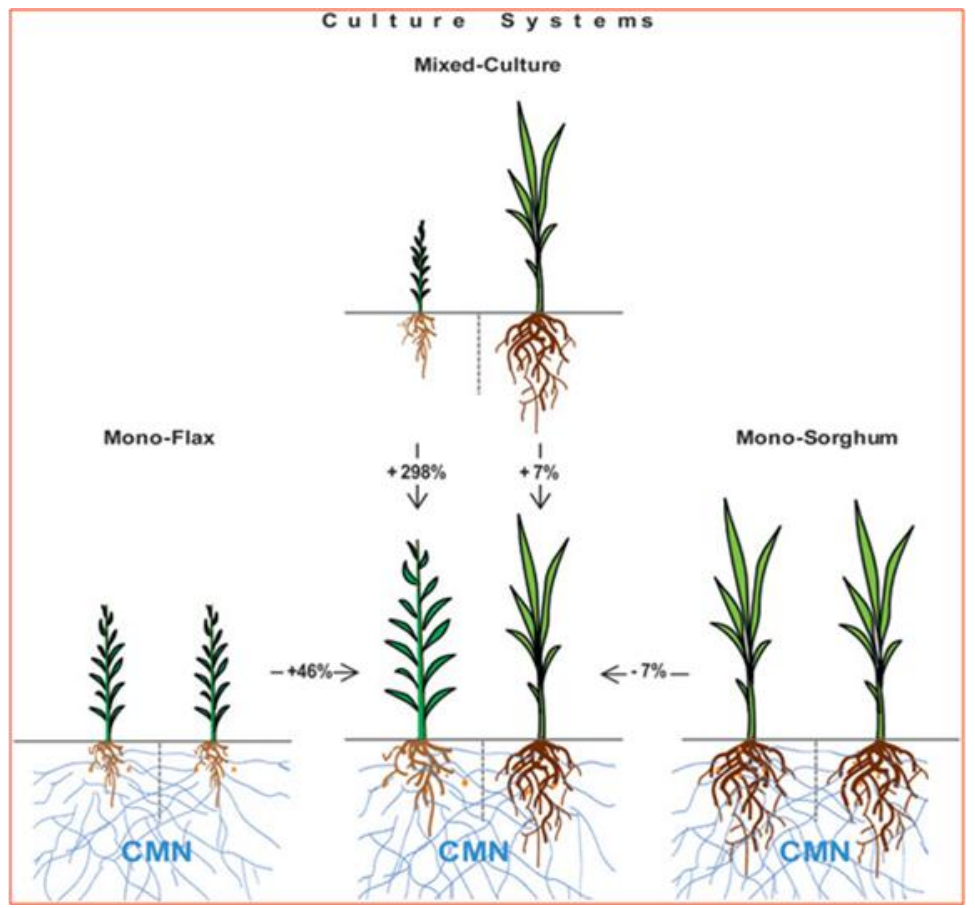

Walder et al.,2010

Fig.15 Stress-related signals from damaged plants, can pass through soil within the plant rhizosphere

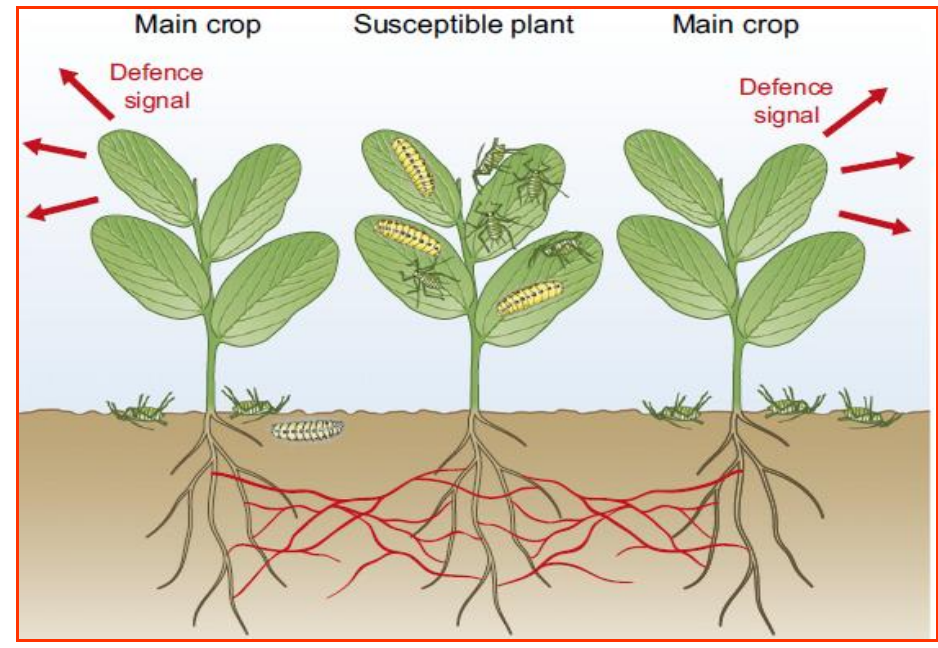

Aphids drain plants of carbon, severely damaging them and can decrease mycorrhizal colonization of bean plants by $20 \%$ (Babikova et al., 2013). Therefore, it might benefit AM fungi to signal a warning to their host plants so that they can then repel or limit attack by aphids, thus maintaining carbon flow to the fungus.
Figure 12 explains the four Hypothetical scenarios in which Signal transfer via a CMN is modulated in order to acquire the most benefit for the fungus.

A: The fungus preferentially allocates signals to plants that are of most immediate value to it in terms of the quantity of carbon that the 
fungus gains from the plants, thereby protecting its current most important carbon source.

B: Signals are preferentially allocated to plants that elicit the strongest defence response, and hence are better at repelling herbivores and attracting the herbivore's enemies via induced volatiles.

C: CMNs are formed between different plant phenotypes so the fungus can "hedge its bets" against the scenario of loss of phenotypes; by warning a wide variety of phenotypes, there is a greater likelihood that one of them remains healthy, thereby maintaining carbon flow to the fungus.

D: The fungus may withhold signals to plants that harbour extensive colonization by other competing fungi. If signaling is costly to the fungus, it might be predicted that the fungus gains greater benefit by signaling preferentially to a plant that is colonized mainly by itself, rather than to a plant that is colonized mainly by its competitors.

\section{Why plant use CMN for signal transfer?}

For below ground communication to be effective, enough of the infochemical/ signal molecule must be produced by the 'sending' organism to get through the soil matrix and reach the 'receiving' organism in large enough concentrations to trigger a response.

Once signal molecule reach the soil, whether through root exudation or by leaching during decomposition, available concentrations quickly decline due to biotic and abiotic degradation, sorption to organic matter, and the formation of complexes with metals. Such mechanisms create a substantial barrier to the reception of bioactive levels of signal molecule by receiving organisms. So, to prevent the signal molecule from degradation plants use CMN as an effective media to send signals to receiver plants.

\section{Speed of signal transfer}

The signal (and the response to the signal) must be rapid enough to successfully deter herbivores before they can travel between the infested donor and the uninfested receiver plants. Johnson and Gilbert (2017) combined the evidence from the studies to date of the speed of the signal and response, i.e., the time from pest or pathogen addition in the donor to the response detected in the receiver plants, and concluded that the signal was detected, in terms of measurable response by receiver plants, within $24 \mathrm{~h}$ of the donor receiving the pest. Peak response in receivers occurred between 48 and $100 \mathrm{~h}$ after infestation of the donor (Fig. 13). These findings suggest that the CMN-based pest warning signals are indeed likely to be of importance to pestplant-fungal dynamics, opening the field for further research into the wider ecological and evolutionary consequences of this mode of plant-plant communication. The length of time over which the signals are effective (i.e., how long the plants produce the defence response) is so far unknown, but this is also likely to affect strongly the wider ecosystem impact of CMN based signalling.

\section{Implications to agriculture}

The presence of a CMN strongly enhanced the biomass production of flax in mixed culture with sorghum. Sorghum was not significantly affected by the presence of a $\mathrm{CMN}$ in the mixed culture with flax. The flax plants grew significantly faster in the flax/sorghum mixed cultures than in the flax monocultures. Conversely, the growth of sorghum was only marginally influenced by the culture system. Flax invested little carbon but gained up to $94 \%$ of the nitrogen and phosphorus provided by the $\mathrm{CMN}$, which 
highly facilitated growth, whereas the neighbouring sorghum invested massive amounts of carbon with little return but was barely affected in growth. Overall biomass production in the mixed culture surpassed the mean of the two monocultures. Thus, CMNs may contribute to interplant facilitation and the productivity boosts often found with intercropping compared with conventional monocropping (Fig.14).

Stress-related signals from damaged plants, for example elicited by herbivore attack, can pass through soil within the plant rhizosphere and, more effectively, via shared arbuscular mycorrhizal fungal networks (see rhizosphere connections in red) to intact plants. These cause induction of volatile defence signals repelling herbivores and attracting parasitoids to attack the herbivores. This opens up the possibility of using susceptible plants within the main crop so that, when attacked, susceptible plants signal via mycorrhizal rhizosphere connections to the main crop. This would then mount defence when needed, rather than suffering the metabolic cost of constitutive defence normally provided by resistant crop plants (Fig. 15).

\section{Signal is not only chemical but also electrical}

Herbivore-induced early defence response in plants involves cytosolic $\mathrm{Ca}_{2}^{+}$spiking, leading to changes in electrical potential on the surface of the cell followed by an electrical signal (action potential). The speed of this electrical signal is much faster $(40 \mathrm{~m} / \mathrm{s})$ than transfer of chemical signals via vascular tissues, and the signal travels through the entire plant from the point of perceived input. The electrical signals activate biosynthesis of jasmonate in distal leaves and the genes involved in transmission of these signals were recently identified in Arabidopsis (Mousavi et al., 2013).
If the electrical signal reaches sites of mycorrhizal colonization, and if it can continue beyond via the CMN, then this could potentially lead to the development of novel electrical crop protection treatments.

Pesticides have helped hugely to increase crop yields throughout the 20th century, there is now an increasing need to find alternative sustainable ways to maintain and improve food security. Due to the growing recognition of the negative aspects to pesticide usage that includes environmental pollution, human health issues, killing beneficial arthropods such as pollinators and natural enemies of pests and the development of acquired resistance to many pesticides by the pests. Therefore, there is now a growing body of research into integrated pest management to encourage more sustainable crop management. CMN-based plant-plant signals could be part of this toolkit. Clearly, for crops to self-protect in this way, CMNs need to connect the individual crop plants. Since deep ploughing and other forms of tilling destroy the fungal networks, this type of pest control could operate only in minimal till or no till systems, including perennials. Further research is needed on the extent to which soil management aids or inhibits CMN formation and signal transfer. It would also be essential to understand which crop species can send or receive signals, how far signals can travel and through how many individual crop plants. CMN-based plant-plant signals warn plants before the pest attack, there may be scope for developing these signals as part of the toolkit in the fight against agricultural pests and disease.

\section{References}

Babikova, Z., Johnson, D., Bruce, T., Pickett, J., and Gilbert, L., 2013, Underground allies: How and why do mycelial 
networks help plants defend themselves? Bioessays, 35: 21-26

Bago, B., Zipfel, W., Williams, R., M., Jun, J., Arreola, R., Lammers, P., J., and Shachar-Hill, Y., 2002, Translocation and utilization of fungal storage lipid in the arbuscular mycorrhizal symbiosis. Pl. Physiol. 128: 108-124.

Barto, K., E., Hilker, M., M€Uller, F., Mohney, F., Weidenhamer, J., D., and Rillig, M., C., 2011, The fungal fast lane: common mycorrhizal networks extend bioactive zones of allelochemicals in soils. PLoS One, 6: 271-279.

Chamberlain, K., Guerrieri, E., Pennacchio, F., Pettersson, J., Pickett, J., A., Poppy, G., M., and Woodcock, C., M., 2001, Can aphid-induced plant signals be transmitted aerially and through the rhizosphere? Biochem. Sys. Ecol. 29: 1063-1074.

Dicke, M., and Dijkman, H., 2001, Withinplant circulation of systemic elicitor of induced defence and release from roots of elicitor that affects neighbouring plants. Biochem. Sys. Ecol. 29: 10751087.

Gilbert, L., and Johnson, D., 2017, Plant-Plant communication through common mycorrhizal networks., Adv. Bot. Res., 82: 83-95

Grelet, G., A., Johnson, D., Vrålstad, T., Alexander, I. J., and Anderson, I., C., 2010, New insights into the mycorrhizal Rhizoscyphus ericae aggregate: spatial structure and cocolonization of ectomycorrhizal and ericoid roots. New Phytol., 188: 210-222.
Leake, J., R., Johnson, D., Donnelly, D., P., Muckle, G., Boddy, L., and Read, D., J., 2004, Networks of power and influence: the role of mycorrhizal mycelium in controlling plant communities and agro ecosystem functioning. Can. J. Bot. 82:1016-1045.

Mousavi, A., R., Chauvin, A., Pascaud, F., and Kellenberger, S. 2013, Glutamate Receptor-Like genes mediate leaf-toleaf wound signalling. Nature 500: 422429.

Simard, S., W., Beiler, K., J., Bingham, M., A., Deslippe, J., R., Philip, L., J., and Teste, F., P., 2012, Mycorrhizal networks: mechanisms, ecology and modelling, Fungal Biol.26:39-60.

Simard, S., W., Perry, D., A., Jones, M., D., Myrold, D., D., Durall, D., M., and Molina, R., 1997, Net transfer of carbon between ectomycorrhizal tree species in the field. Nature, 388:579-582.

Song, Y., Y., Zeng, R., S., Xu, J., A., F., Li, J., Shen, X., A., and Yihdego, W., G., 2010, Interplant communication of tomato plants through underground common mycorrhizal networks. PLoS One, 513: 32-38.

Taylor, A., F., S., 2006, Common mycelial networks: life-lines and radical addictions. New Phytol., 169: 6-8.

Walder, F., Niemann, H., Natarajan, M., Lehmann, M., F., Boller, T., and Wiemken, A., 2010. Mycorrhizal networks: common goods of plants shared under unequal terms of trade. Plant Physiol. 159:789-797.

\section{How to cite this article:}

Vanishri, B.R. and Mamata Khandappagol. 2018. Belowground Plant-Plant Interaction by Common Mycorrhizal Network (CMN). Int.J.Curr.Microbiol.App.Sci. 7(09): 1691-1705. doi: https://doi.org/10.20546/ijcmas.2018.709.205 